\title{
Amoebiasis due to free-living amoebae
}

INSERM

\section{Source}

INSERM. (1999). Orphanet: an online rare disease and orphan drug data base.

Amoebiasis due to free-living amoebae. ORPHA:68

Amoebiasis is a parasitosis caused by free-living amoebae that are able to survive in an autonomous state in all natural environments. Some species, belong ing principally to the Acanthamoeba and Naegleria genera are able to parasitize humans. 